\title{
Modelling anisotropic water transport in polymer composite reinforced with aligned triangular bars
}

\author{
BRYAN PAJARITO ${ }^{1,2, *}$, MASATOSHI KUBOUCHI ${ }^{1}$ and SAIKO AOKI ${ }^{1}$ \\ ${ }^{1}$ Department of Chemical Engineering, Tokyo Institute of Technology, 2-12-1 S4-5, \\ Ookayama, Meguro-ku, Tokyo 152 8552, Japan \\ ${ }^{2}$ Department of Chemical Engineering, University of Philippines, Diliman, Quezon City 1101, Philippines
}

MS received 10 August 2012

\begin{abstract}
This work reports anisotropic water transport in a polymer composite consisting of an epoxy matrix reinforced with aligned triangular bars made of vinyl ester. By gravimetric experiments, water diffusion in resin and polymer composites were characterized. Parameters for Fickian diffusion and polymer relaxation models were determined by least-square curve fitting to the experimental data. Diffusion parameters of epoxy and vinyl ester resin were used as input during development of finite element (FE) model of polymer composite. Through transient FE diffusion analysis, anisotropic water transport in thickness direction of the polymer composite was numerically predicted and validated against experimental results. The case of using impermeable triangular bars was also numerically simulated. The diffusivity of reinforced aligned triangular bars was confirmed to affect anisotropic water transport in the composite. The results of this work suggest possible use of polymer composite for barrier and fluid removal applications.
\end{abstract}

Keywords. Anisotropy; diffusion; triangular bar; polymer composite; finite element analysis.

\section{Introduction}

Diffusion and mass transport of fluids in polymer composites are well known to be anisotropic and highly dependent on the orientation of its reinforcement fillers. For instance, when impermeable flake fillers are oriented perpendicular to the diffusion direction, effective transport of fluid into the polymer composite is slower than the unreinforced polymer (Moggridge et al 2003; Lape et al 2004; Chen and Papathanasiou 2007; Cussler 2007). However, when these flakes are oriented parallel to the diffusion direction, the resulting fluid transport behaviour is faster than the perpendicular case (Eitzmann et al 1996; Ly and Cheng 1997; White and Cussler 2006), sometimes faster than the unreinforced polymer (Pajarito et al 2012) due to local accelerated transport at the flake-polymer interphase region. This orthogonal dependency of fluid transport in polymer composites is due to the effect of filler orientation to diffusion resistance. Longer diffusion path length and smaller diffusion area are present in composites reinforced with perpendicular flakes than with parallel flakes. Polymer composites filled with perpendicular impermeable fillers are thus effective materials for barrier applications.

Now consider a polymer matrix reinforced with aligned, impermeable triangular bars as shown in figure 1. Each layer of the assembly is composed of aligned triangular bars

*Author for correspondence (bryan.pajarito@up.edu.ph) set at certain filler spacing. Such a structure, when used as reinforcement for a permeable polymer matrix, is expected to induce anisotropic fluid transport behaviour in the composite. While anisotropic fluid transport in flake polymer composite occurs in perpendicular and parallel orientation of flakes, anisotropic fluid transport in a material reinforced with aligned triangular bars are assumed to be evident in the thickness direction of the polymer composite. Effective fluid transport is hypothesized to be fast when diffusion is initiated at the pointed/sloped side of the triangular bar assembly; and slow when diffusion starts at the flat/base side of the filler assembly. The expected anisotropy in aligned triangular bars is somewhat analogous to the effect of flake orientation in effective diffusion. The exposed matrix area available for diffusion at the pointed/sloped side of the triangular bar assembly is higher than at the flat/base side of the filler assembly.

A possible application of anisotropic fluid transport property in polymer composites reinforced with aligned triangular bars is on barrier and fluid removal processes. Exposing surface of the polymer composite where the flat/base side of the triangular bars is oriented would slow down diffusion and act as a barrier for the diffusing environment. Prolonged exposure of the polymer composite to the diffusing environment would eventually plasticize the matrix and decrease its mechanical properties. Since plasticization is a physical change, its effect could be reversed by fluid removal through drying (Weitsman 2012). Exposing surface of the polymer composite where the pointed/sloped side of the triangular bars is oriented would enhance the drying rate and fluid removal from the material. 


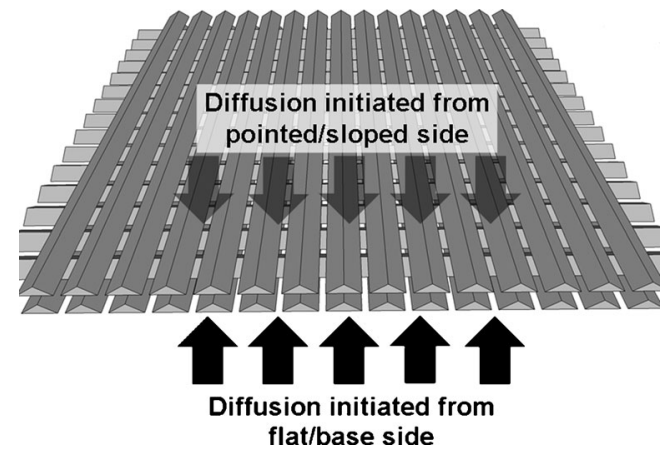

Figure 1. Assembly of aligned triangular bars.

This work reports anisotropic fluid transport in polymer composite reinforced with aligned triangular bars. Water was chosen to represent the diffusing fluid medium, while acid anhydride-cured epoxy was used as polymer matrix. Due to difficulty in making triangular bars made of impermeable material such as glass, cured vinyl ester was used due to its low water absorption capacity compared to epoxy. Gravimetric immersion tests were performed and transport parameters were determined from experimental results. Transient water transport in polymer composites was numerically simulated by the finite element (FE) method using material input from resin transport properties. The case of an epoxy matrix reinforced with triangular bars made of impermeable material was also simulated and reported.

\section{Experimental}

The polymer matrix of composite specimens was made from alicyclic type epoxy resin (ECR-1105, Sumitomo Bakelite Co., Ltd.) cured with stoichiometric amount of methyltetrahydrophthalic anhydride (ECH-202, Sumitomo Bakelite Co., Ltd.) for $3 \mathrm{~h}$ at $80{ }^{\circ} \mathrm{C}, 2 \mathrm{~h}$ at $100{ }^{\circ} \mathrm{C}$ and $5 \mathrm{~h}$ at $120{ }^{\circ} \mathrm{C}$ curing steps. The triangular bars having length of $10 \mathrm{~mm}$, base of $5 \mathrm{~mm}$ and height of $1.6 \mathrm{~mm}$ were pre-cured with vinyl ester resin (Ripoxy R-804, Showa Highpolymer Co., Ltd.) specially molded for $2 \mathrm{~h}$ at $50{ }^{\circ} \mathrm{C}$ and $3 \mathrm{~h}$ at $100{ }^{\circ} \mathrm{C}$ curing steps. The pre-cured triangular bars were manually assembled in a layer-wise fashion guided by a pattern laid out in $1 \mathrm{~mm}$ grid paper. The spacing between bars was set at $1 \mathrm{~mm}$. The bars were bonded at their ends using cyanoacrylate adhesive (Aron Alpha Extra 2000, Toagosei Co., Ltd.). The complete assembly was composed of 90 triangular bars with each layer consisting of 15 aligned fillers. The assembly was then fixed inside a metal square mold, where the degassed epoxy matrix solution was poured in. After curing in a thermal press, the final polymer composite specimen was cut into $100 \times 100 \mathrm{~mm}$ square plate. The thickness was $\sim 10 \mathrm{~mm}$ and the edges were sealed with stainless steel tape to ensure through-the-thickness direction of water diffusion in the polymer composite specimen. Two types of polymer composite specimens were prepared as shown by their crosssection in figure 2. In specimen A, pointed/sloped side of

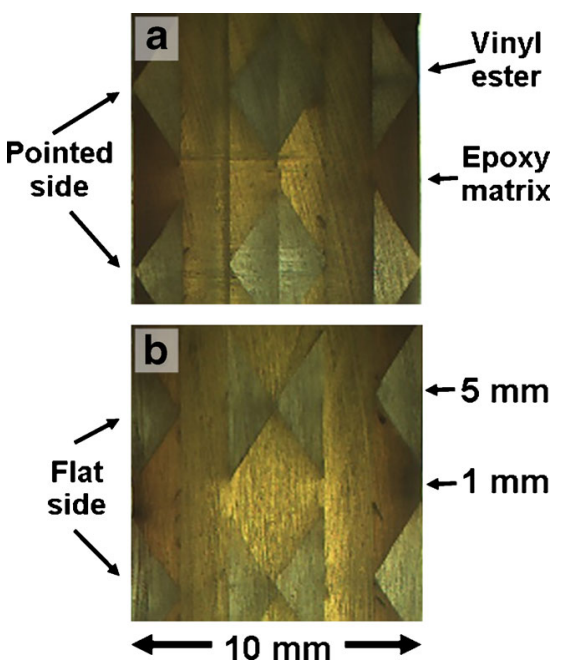

Figure 2. Cross-sectional view of polymer composite specimens $\mathbf{a}$ and $\mathbf{b}$.

the triangular bar assembly was oriented at the surface of the composite. In specimen B, flat/base side of the triangular bar assembly was exposed at the surface of the composite. Pure epoxy and vinyl ester specimens were also prepared for the experiment.

After determining the weight increase due to applied adhesive and stainless steel tape at the edges, oven-dried specimens were immersed in de-ionized water inside rectangular glass containers. Teflon holders were used to avoid physical contact among specimens during water immersion. The rectangular glass containers were covered with plastic sheets to avoid loss of immersion water due to evaporation. The temperature of immersion was kept at $80{ }^{\circ} \mathrm{C}$ for $1200 \mathrm{~h}$ using water bath. During immersion, weight of specimens was monitored to determine the percent water uptake relative to the initial dry weight. The water uptake content $\left(M_{\mathrm{t}}\right)$ absorbed by specimen was calculated from its weight before $\left(w_{0}\right)$ and after $\left(w_{\mathrm{t}}\right)$ exposure as follows:

$$
M_{t}=100 \times\left(\frac{w_{\mathrm{t}}-w_{0}}{w_{0}}\right) .
$$

Three test specimens were prepared for each specimen condition and the average $M_{\mathrm{t}}$ was reported during water immersion.

\section{Results and discussion}

After $1200 \mathrm{~h}$ of immersion in de-ionized water at $80{ }^{\circ} \mathrm{C}$, the average water uptake curves of resin and polymer composite specimens are presented in figure 3 , where $M_{\mathrm{t}}$ is plotted vs $\sqrt{ } t$ to show the initial linear diffusion curve. Water uptake of specimens increases abruptly for the initial part of all uptake curves. Vinyl ester resin specimen, VE, reached the water saturation level within $1200 \mathrm{~h}$ of exposure. Water uptake curves of epoxy resin, EP and composite specimens, $\mathrm{A}$ and $\mathrm{B}$, consist of the initial linear diffusion plot followed 


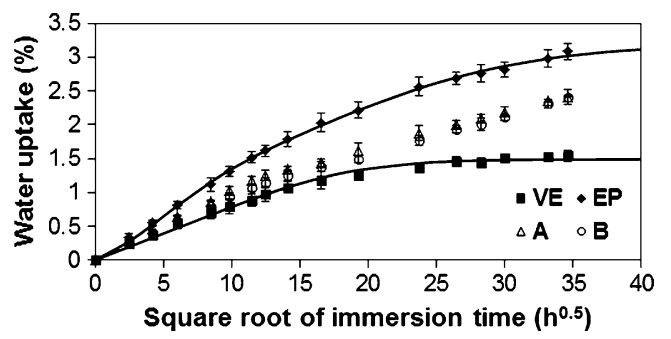

Figure 3. Water transport process of resin and polymer composite specimens at $80^{\circ} \mathrm{C}$.

by second slow-rising stage, where more water content is slightly absorbed by the specimen. The second slow-rising stage of water uptake in composite specimens A and B are somewhat similar to the epoxy specimen EP. For most part of the immersion, water uptake of composite specimen $\mathrm{A}$ is slightly higher than the composite specimen B.

To model water transport in the specimens, numerous diffusion models have been proposed and used in the literature. Researchers frequently use one-dimensional Fickian model to describe water diffusion in polymeric materials (Crank 1975). It is assumed that the polymer specimen plate is infinite and water only diffuses in through-the-thickness direction, which is proportional to the concentration gradient of the material. The stainless steel tape bonded at the edge surfaces of the specimens prevents water from being absorbed at the edges and forces water to diffuse one-dimensionally through the thickness of specimens. For a plate of thickness $2 h$, Fickian model expresses $M_{\mathrm{t}}$ as a function of immersion time $t$ :

$$
M_{\mathrm{t}}=M_{\infty}\left\{1-\frac{8}{\pi^{2}} \sum_{n=0}^{\infty} \frac{1}{(2 n+1)^{2}} \exp \left[-\frac{D(2 n+1)^{2} \pi^{2} t}{4 h^{2}}\right]\right\} \text {, }
$$

where $M_{\infty}$ is the maximum equilibrium amount of absorption and $D$ the Fickian diffusion coefficient in the thickness direction, which is supposed to be constant and independent of spatial and temporal coordinates. Fickian diffusion process in $M_{\mathrm{t}}$ vs $\sqrt{ } t$ curve is characterized by initial linear plot followed by a saturation level, as exemplified by specimen $\mathrm{VE}$ in figure 3. To get accurate value of Fickian diffusion coefficient $D$ and apparent equilibrium amount of absorption $M_{\infty}$ for specimen, VE, the analytical model (2) was employed to fit experimental data points by the least-square method. A script written in GNU octave utilizing its leasqr function facilitated the data fitting. After analytical curve fitting, Fickian diffusion parameters of specimen VE were obtained and listed in table 1 and the analytical curve was plotted in figure 3.

As shown by the data points of epoxy resin EP in figure 3, typical Fickian diffusion is not suitable for describing the whole water transport process. Deviation from Fickian diffusion is usually attributed to polymer relaxation (Crank 1975), a long-term behaviour where polymer molecules are
Table 1. Water transport parameters of resin specimens VE and EP.

\begin{tabular}{ccccc}
\hline & $D\left(\mathrm{~mm}^{2} / \mathrm{h}\right)$ & $M_{\infty, \mathrm{F}}(\%)$ & $k\left(\mathrm{~h}^{-1}\right)$ & $M_{\infty, \mathrm{R}}(\%)$ \\
\hline $\mathrm{VE}$ & 0.55 & 1.487 & - & - \\
$\mathrm{EP}$ & 0.021 & 2.624 & 0.019 & 0.563 \\
\hline
\end{tabular}

stretched due to the presence of diffusing water molecules. Polymer relaxation results in redistribution of voids and free volumes and more absorption of water content in the polymer. A more versatile model (Berens and Hopfenberg 1978) was proposed to model transport in polymers, where a linear superposition of independent contributions from Fickian diffusion and polymer relaxation were involved. For epoxy resin, EP, the total amount of absorption, $M_{\mathrm{t}}$, at immersion time $t$ is expressed as:

$$
M_{\mathrm{t}}=M_{\mathrm{t}, \mathrm{F}}+M_{\mathrm{t}, \mathrm{R}},
$$

where $M_{\mathrm{t}, \mathrm{F}}$ and $M_{\mathrm{t}, \mathrm{R}}$ are the contributions of Fickian and relaxation processes, respectively at immersion time $t$. The contribution due to Fickian process is described by (2) and the contribution due to polymer relaxation induced water absorption is given by

$$
M_{\mathrm{t}, \mathrm{R}}=M_{\infty, \mathrm{R}}[1-\exp (-k t)]
$$

where $k$ is the relaxation-rate constant and $M_{\infty, \mathrm{R}}$ is the ultimate amount of absorption due to polymer relaxation. The total amount of absorption is given by substitution of (2) and (4) into (3) resulting in

$$
\begin{aligned}
M_{\mathrm{t}}=M_{\infty, \mathrm{F}}\{1 & -\frac{8}{\pi^{2}} \sum_{n=0}^{\infty} \frac{1}{(2 n+1)^{2}} \\
& \left.\times \exp \left[-\frac{D(2 n+1)^{2} \pi^{2} t}{4 h^{2}}\right]\right\} \\
& +M_{\infty, \mathrm{R}}[1-\exp (-k t)] .
\end{aligned}
$$

By using (5), curve fitting was conducted to obtain water diffusion parameters of epoxy resin EP as listed in table 1. Figure 3 shows results of curve fitting together with experimental data. As shown, Fickian diffusion dominates the initial linear water absorption stage. After the diffusion curve apparently reaches the first water stable level, the contribution from polymer relaxation governs the second part of the transport process.

\section{Numerical modelling}

Though one-dimensional Fickian diffusion model (2) could be applied to describe fluid transport in polymer composites (Springer 1981; Alvarez et al 2007), the analytical solutions for water transport prediction is initially developed for homogeneous, unreinforced polymeric materials (Crank 1975). To model water transport in polymer composite made of combined different resins, FE method was employed in this 
study to simulate water diffusion process in composite specimens. The finite element grid generator Gmsh (Geuzaine and Remacle 2009) was employed for building FE model of the composite specimen. FE model shown in figure 4 has total dimension of $6 \times 6 \times 5 \mathrm{~mm}$, where dimension, alignment and spacing of triangular bars in FE model are same with the experimental composite specimens. Because of symmetry, only half-thickness of the experimental composite specimens was modelled. Furthermore, the developed FE model is periodic in $x$ and $y$ directions, where surface concentration on one edge of the model is set equal to the surface concentration on the opposite side. The water transport properties obtained for resin specimens VE and EP (see table 1) were used as input parameters for material properties in FE model. The typical Fickian diffusion was considered for the vinyl ester triangular bar regions in FE model, while polymer relaxation parameters were additionally included for modelling of the epoxy matrix region. To simulate water transport in composite specimen A, the top surface of FE model, which was exposed to water immersion during experiment of composite specimen A, was set to be fully water saturated as the boundary condition of FE model. To simulate water transport in composite specimen B, the bottom surface of FE model was set to be fully water saturated. The initial volume concentration of water in FE model was set to zero.

After constructing FE model and applying the appropriate boundary and initial conditions, the moisture concentration distributions in FE model were calculated as function of immersion time. A maximum immersion time of $1200 \mathrm{~h}$ was set during transient diffusion simulation, which is similar to the water immersion experiment. The general finite element solver GetDP (Dular et al 1999) was employed for water diffusion analysis. GetDP requires the symbolic weak formulation of problems for input. The weak formulation of Fick's second law of diffusion is given as (Kwon and Bang 1997):

$$
-\int_{\Omega} D \nabla \phi \cdot \nabla \omega \mathrm{d} \Omega+\int_{\Gamma} D \frac{\partial \phi}{\partial n} \omega \mathrm{d} \Gamma-\int_{\Omega} \nabla_{t} \phi \omega \mathrm{d} \Omega=0,
$$

in which $\phi$ is the normalized water concentration and $\omega$ is a weighting function. FE model consists of the epoxy and

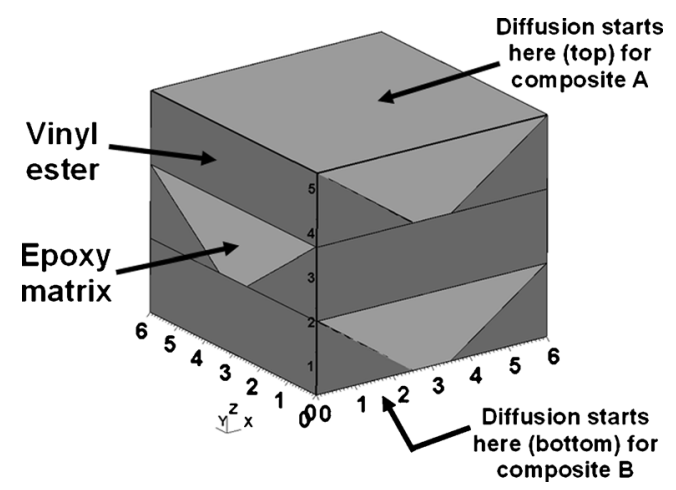

Figure 4. FE model of polymer composite specimens A and B. vinyl ester regions, $\Omega=\left\{\Omega_{\mathrm{EP}}, \Omega_{\mathrm{VE}}\right\}$ and boundary surfaces, $\Gamma$. On the other hand, the weak formulation for the problem of polymer relaxation (Berens and Hopfenberg 1978) is expressed as

$$
\int_{\Omega} k(1-\phi) \omega \mathrm{d} \Omega-\int_{\Omega} \nabla_{\mathrm{t}} \phi \omega \mathrm{d} \Omega=0 .
$$

In this problem, the normalized water concentration is only a function of immersion time $t$ and only applicable to epoxy region $\Omega_{\mathrm{EP}}$. After transient diffusion simulation, the computed normalized water concentration distribution was integrated over FE model to get the water absorption content $M_{\mathrm{t}}$ at every time $t$ :

$$
M_{\mathrm{t}}=F \cdot M_{\infty} \int_{\Omega} \phi \mathrm{d} \Omega,
$$

where the constant $M_{\infty}$ of epoxy and vinyl ester resin specimens are given for diffusion and polymer relaxation in table 1 and the factor $F$ is the volume ratio of composite specimen $(100 \times 100 \times 10 \mathrm{~mm})$ to FE model $(6 \times 6 \times 5 \mathrm{~mm})$.

Figure 5 shows comparison of $\mathrm{FE}$ analysis results with experimental data for water transport in composite specimens $\mathrm{A}$ and $\mathrm{B}$ at $80{ }^{\circ} \mathrm{C}$. During the initial diffusion stage of composites $\mathrm{A}$ and $\mathrm{B}, \mathrm{FE}$ results agree well with the water absorption content measured experimentally during immersion. Significant deviation between the experimental data points and FE results takes place after $\sim 100 \mathrm{~h}$, where composite specimens have lower experimental water uptake than FE prediction. Beside from epoxy matrix and vinyl ester triangular bars, the composite specimens also contain cyanoacrylate adhesive, which was used to bond the triangular bars at their ends during specimen fabrication. During weight recording of wet composite specimens, visual inspection of specimen surface revealed partial matrix dissolution near the edges, where the cyanoacrylate adhesive was applied. The partial dissolution of matrix during water immersion lowers water content absorption of composite specimens.

Though FE model and experimental water immersion both showed water transport in composite A different to composite $\mathrm{B}$, FE model predicts water content absorption in composite B higher than composite A. It is hypothesized earlier that water transport in composite $\mathrm{A}$ is faster than composite

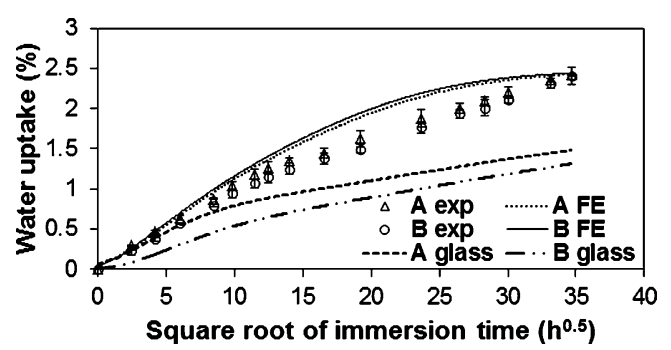

Figure 5. Comparison of FE results and experimental data. 
B due to availability of exposed matrix area. To explain this, figure 6 shows water concentration distribution in FE model cross-section of polymer composites A and B after $100 \mathrm{~h}$ of immersion. While water absorption capacity of vinyl ester is lower than epoxy, water diffusion coefficient of vinyl ester is higher than epoxy. Exposing the base side of vinyl ester triangular bars to diffusion reveals more available area for diffusion than exposing the pointed side. This results in a slightly faster water transport in FE model of polymer composite B than polymer composite A as shown.

The case of an epoxy matrix reinforced with aligned triangular bars made of impermeable material such as glass was also numerically modelled. The input material properties of triangular bars in FE model was set as: $M_{\infty}=0$ and $D=0$. The input material properties of epoxy matrix are shown in table 1 . Figure 5 shows predicted water content absorption of FE model of epoxy composites A and B reinforced with glass triangular bars. Figure 6 shows water concentration distribution in FE model cross-section. If the triangular bars are impermeable to water, water content absorption in polymer composite $\mathrm{A}$ is numerically estimated to be significantly higher than polymer composite B. This is in accordance with the earlier assumption of slow transport from flat side and fast transport from pointed side of the triangular bar assembly. The difference of water content absorption in FE model of composite A from composite B describes

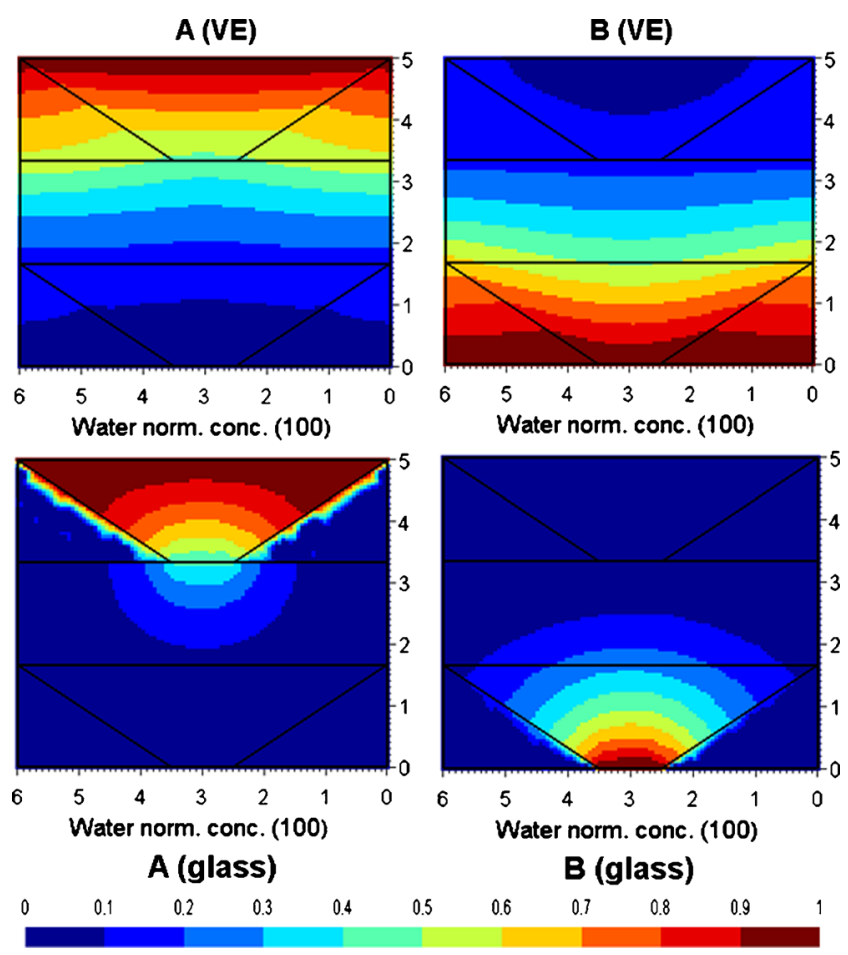

Figure 6. Water concentration distribution in FE model crosssection of polymer composites A and B reinforced with vinyl ester (VE) and impermeable material (glass) after $100 \mathrm{~h}$ of immersion at $80^{\circ} \mathrm{C}$. anisotropy of water transport in the composite. The diffusivity of reinforced aligned triangular bars in the composite was confirmed to affect anisotropic water transport in the material.

\section{Conclusions}

By water immersion gravimetric experiments, anisotropic water transport at $80{ }^{\circ} \mathrm{C}$ in epoxy composites reinforced with aligned vinyl ester triangular bars was studied. Only vinyl ester resin specimen reached water saturation level during $1200 \mathrm{~h}$ immersion. One-dimensional Fickian diffusion analytical model was employed to fit the experimental data of vinyl ester resin specimen by the least-square method. For the case of epoxy resin specimen, a versatile diffusion model, which takes into account Fickian diffusion and polymer relaxation were utilized for curve fitting. Subsequently, water transport parameters of both resin specimens were determined. To model water transport in polymer composite specimens during immersion at $80^{\circ} \mathrm{C}$, FE method was used. FE model of the composite specimens was built and transient water transport was numerically simulated. The matrix and triangular bar regions of FE model used the water transport properties of epoxy and vinyl ester resins as input during simulation. Numerical predictions of water content absorption by FE model were consistent with experimental water content absorption of composite specimens. For reinforced semipermeable triangular bars such as vinyl ester, FE model predicts water transport to be slightly faster from the flat side of the composite than from the pointed side. If the triangular bars are made of materials impermeable to water such as glass, FE model predicts water transport to be significantly faster from the pointed side of the composite than from the flat side. The dependence of water content absorption to which side of the polymer composite diffusion initiates is characteristic of anisotropic water transport in the material.

\section{Acknowledgements}

The authors would like to thank Hitachi Scholarship Foundation for financial support and Dr Hideki Sembokuya for fabricating the special metal molds of vinyl ester triangular bars.

\section{References}

Alvarez V, Vazquez A and de la Osa O 2007 J. Compos. Mater. 41 1275

Berens A R and Hopfenberg H B 1978 Polymer 19489

Chen X and Papathanasiou T 2007 J. Plast. Film. Sheet. 23319

Crank J 1975 The mathematics of diffusion (Oxford: Clarendon Press)

Cussler E L 2007 Diffusion Fundamentals 672.1

Dular P, Geuzaine C, Genon A and Legros W 1999 IEEE T. Magn. 351682

Eitzmann D, Melkote R and Cussler E L 1996 AIChEJ 422 
Geuzaine C and Remacle J F 2009 Int J. Numer. Meth. Eng. 791309 Kwon Y W and Bang H 1997 Finite element method using matlab (Boca Raton: CRC Press LLC)

Lape N, Nuxoll E and Cussler E L 2004 J. Membr. Sci. 23629

Ly Y and Cheng Y 1997 J. Membr. Sci. 133207

Moggridge G D, Lape N, Yang C and Cussler E L 2003 Prog. Org. Coat. 46231
Pajarito B, Kubouchi M, Tomita H and Sakai T 2012 Mater. Sci. Tech. Jpn. 4932

Springer G S ed. 1981 Enviromental effects on composite materials (Technomic Publishing Co.)

Weitsman Y J 2012 Fluid effects in polymers and polymeric composites (New York: Springer)

White J and Cussler E L 2006 J. Membr. Sci. 278225 